\title{
Pulmonary ossification syndrome in a patient with chronic thromboembolic pulmonary hypertension
}

\author{
Noreen Amirali Rajwani MD, Lakshmi Puttagunta MD, James Barrie MD FRCPC, Meena Kalluri MD
}

\begin{abstract}
NA Rajwani, L Puttagunta, J Barrie, M Kalluri. Pulmonary ossification syndrome in a patient with chronic thromboembolic pulmonary hypertension. Can Respir J 2014;21(2):83-85.

Pulmonary ossification syndrome is a rare disease characterized by bone tissue formation in the lung parenchyma with or without bone marrow elements. This disorder, often under-recognized, can be idiopathic or secondary to an underlying chronic disorder such as chronic thromboembolic pulmonary hypertension. The pathogenesis involves tissue injury and an alkaline environment in which the precipitation of calcium salts enables alkaline phosphatase activity. This activates profibrogenic cytokines that convert fibroblasts into osteoblasts. Diagnosis can prove challenging and is based on clinical, radiographic and functional tests, and by tissue biopsy. Achieving an accurate and timely diagnosis is essential to avoid erroneous treatments due to misdiagnosis and to expand knowledge of its progression, prognosis and treatment. As the population and prevalence of chronic lung disease increases, it is likely that physicians will encounter more cases of pulmonary ossification.
\end{abstract}

Key Words: Pulmonary hypertension; Pulmonary ossification syndrome

Learning objectives

1. Recognize the two types of pulmonary ossification syndrome.

2. Understand the various imaging modalities and lung function tests that help diagnose pulmonary ossification syndrome.

CanMEDS Competency: Medical Expert

Pretest

1. What is the etiology of pulmonary ossification syndrome?

2. What is the pathogenesis by which chronic thromboembolic pulmonary hypertension causes pulmonary ossification syndrome?

Dulmonary ossification syndrome is a rare disease characterized by bone tissue formation in the lung parenchyma with or without bone marrow elements. The disorder can be idiopathic or secondary to an underlying chronic disorder. A patient who presented with severe acute-onset dyspnea on exertion in light of a history of bilateral unprovoked pulmonary emboli is reported.

\section{CASE PRESENTATION}

A 51-year-old man presented with a two-week history of progressively worsening dyspnea on exertion graded as Medical Research Council 4. Pertinent history included a new dry cough. The patient also exhibited a low-grade fever but no other constitutional symptoms.

Medical history was significant for bilateral unprovoked pulmonary emboli four years before presentation, which were treated with two inferior vena cava filters due to congenital inferior vena cava duplication. The medical history included Crohn disease on immunosuppression, gastrointestinal bleeds and juvenile dermatomyositis.

Physical examination revealed mild respiratory distress, with an oxygen saturation of $91 \%$ at rest and $88 \%$ on exertion. All other vital signs were normal. Auscultation of the lungs revealed expiratory wheezes greater on the right side. The cardiac examination was benign without any extra cardiac heart sounds, pedal edema or elevated jugular venous pressure.

\author{
Le syndrome d'ossification pulmonaire chez un \\ patient atteint d'hypertension pulmonaire \\ thromboembolique chronique
}

Le syndrome d'ossification pulmonaire est une maladie rare caractérisée par la formation de tissus osseux dans le parenchyme pulmonaire, avec ou sans éléments de moelle osseuse. Ce trouble, souvent sous-diagnostiqué, peut être idiopathique ou secondaire à une maladie chronique sous-jacente, telle que l'hypertension pulmonaire thromboembolique chronique. La pathogenèse inclut une lésion tissulaire et un milieu alcalin qui, à cause de la précipitation des sels de calcium, stimule l'activité de la phosphatase alcaline. Ce phénomène active les cytokines profibrogènes, qui convertissent les fibroblastes en ostéoblastes. Le diagnostic, qui se fonde sur des tests cliniques, radiographiques et fonctionnels et sur une biopsie des tissus, peut se révéler difficile à poser. Il est essentiel de poser un diagnostic exact et rapide pour éviter les traitements erronés causés par un mauvais diagnostic et pour accroître les connaissances relativement à sa progression, à son pronostic et à son traitement. Puisque la population et la prévalence des maladies pulmonaires chroniques augmentent, il est probable que les médecins observent plus de cas d'ossification pulmonaire.

His arterial blood gas showed a $\mathrm{pH}$ of 7.45 , a $\mathrm{pCO}_{2}$ of $33 \mathrm{mmHg}$, a $\mathrm{pO}_{2}$ of $54 \mathrm{mmHg}$ and an $\mathrm{HCO}_{3}$ level of $22.5 \mathrm{mmol} / \mathrm{L}$. Laboratory investigations showed a white blood cell count of $15.1 \times 10^{9} / \mathrm{L}$; a mild microcytic anemia. He was shown to be hypogammaglobulinemic with low levels of immunoglobulin (Ig) G (2.04 g/L), IgA (0.62 g/L) and IgM $(0.46 \mathrm{~g} / \mathrm{L})$.

A computed tomography (CT) scan of the chest showed a chronic reticulonodular interstitial pattern bilaterally in the lower lobes (Figure 1). Calcified nodules were apparent in the right middle lobe on CT bone views (Figure 2). No emboli were identified; however, the central pulmonary arteries were mildly enlarged.

Pulmonary function testing revealed a forced vital capacity (FVC) of $68 \%$, forced expiratory volume in $1 \mathrm{~s}\left(\mathrm{FEV}_{1}\right)$ of $65 \%$ and an $\mathrm{FEV}_{1}$ to FVC ratio of $77 \%$. The total lung capacity was decreased at $68 \%$ and an adjusted diffusing capacity of carbon dioxide (DLCO) was $34 \%$. Overall, this showed a restrictive lung process with a disproportionately low diffusion capacity. A ventilation perfusion scan revealed bilateral mismatched perfusion defects. Contrast echocardiogram showed normal ventricular function and no signs of pulmonary hypertension. A subsequent right heart catheterization showed an elevated pulmonary arterial pressure of $33 \mathrm{mmHg}$ and a normal pulmonary capillary wedge pressure consistent with moderately elevated pulmonary pressures. The present case also demonstrated an increased Tc-99m hydroxymethylene tracer uptake in the lung fields bilaterally.

The patient then underwent a video-assisted thoracoscopic surgeryguided lung biopsy, which showed numerous foci of dystrophic nodular calcification with minute foci of subpleural fibrosis and minimal interstitial inflammation without honeycombing (Figure 3). There was no evidence of venous congestion or iron deposition. There was no evidence of granulomas or malignancy. The biopsy also showed some old recanalized thrombi and dilated pulmonary arteries. Based on all of the above investigations, his diagnosis was diffuse pulmonary ossification (DPO) secondary to chronic thromboembolic pulmonary hypertension.

He was started on anticoagulation, supplemental oxygen and referred to the pulmonary rehabilitation program. Six months after diagnosis, he continues to do well with stable lung function.

University of Alberta, Edmonton, Alberta

Correspondence: Dr Noreen Amirali Rajwani, 3-126A CSB, 11350-83 Avenue, Edmonton, Alberta T6G 2G3. Telephone 780-492-6514,

fax 780-492-4483, e-mail noreen.rajwani@ualberta.ca 


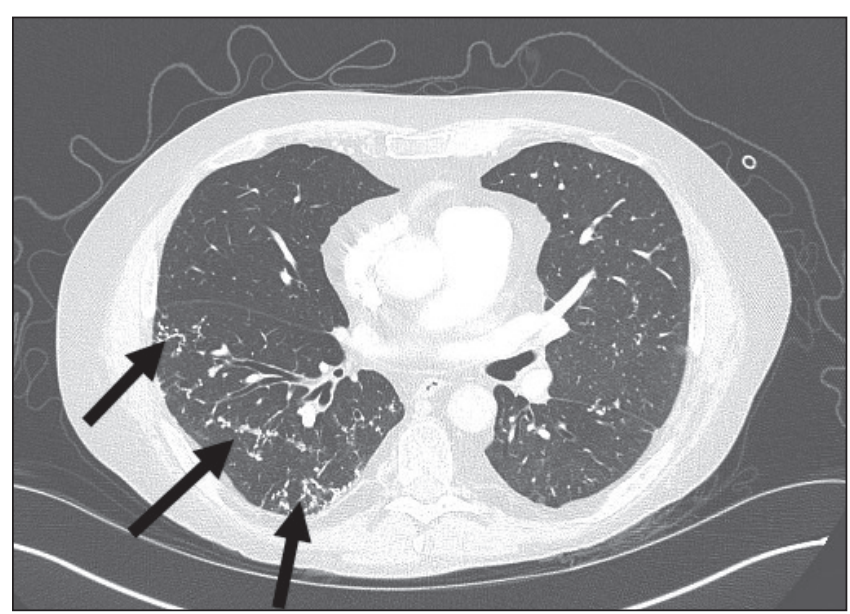

Figure 1) Black arrows indicate the reticulonodular pattern apparent in bilateral lung zones

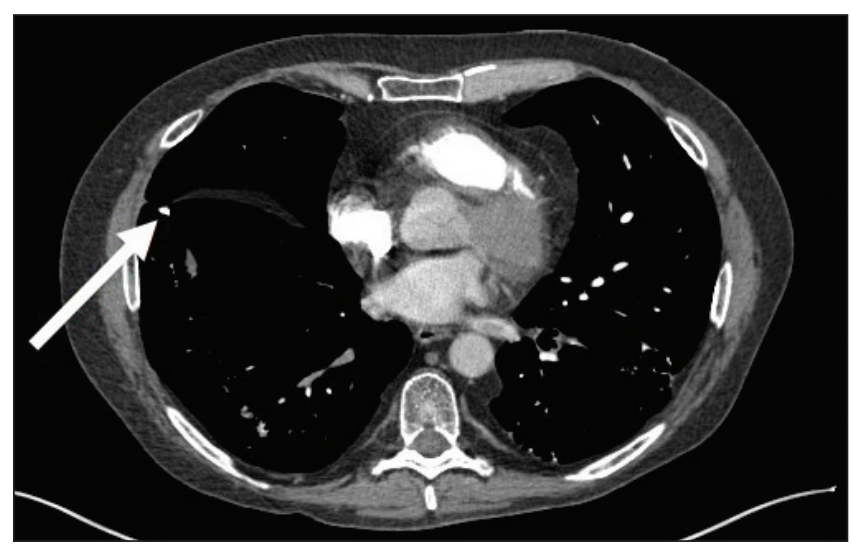

Figure 2) Computed tomography bone view, in which the white arrow indicates calcification of a right middle lobe nodule

\section{DISCUSSION}

$\mathrm{DPO}$ is a rare disease entity that was first described by Lushka in 1856 (1). DPO has most often been found in men $>60$ years of age but can occur in the young and in women (2). Most of the reported cases were recognized postmortem (3). It is a rare disease with variable presentation that can be highly insidious. Most often, it is asymptomatic with subtle imaging changes that can go unrecognized or it can present as progressive dyspnea. Two forms of DPO described based on histological findings are dendritic and nodular; however, distinguishing between the two forms does not alter clinical significance (1).

$\mathrm{DPO}$ can be idiopathic or can be caused by a variety of pulmonary diseases such as infections, granulomatous disease, amyloidosis, metastases, pulmonary fibrosis, adult respiratory distress syndrome, asbestos exposure, busulfan and cystic fibrosis (4). Cardiac causes include mitral stenosis and left ventricular failure (4). One of the causes of DPO is chronic thromboembolic disease with pulmonary hypertension, with our patient meeting all of the diagnostic criteria.

The pathophysiology of DPO is not well known. However, it is believed that tissue injury is the most important provoking factor such that, in an alkaline environment, it initiates the precipitation of calcium salts, enables alkaline phosphatase activity and activates profibrogenic cytokines (5). DPO is believed to cause metaplasia of pulmonary fibroblasts into osteoblasts as a response to a chronic insult and in the setting of chronic pulmonary venous hypertension; repetitive intra-alveolar hemorrhage is probably the stimulus that transforms fibroblast into osteoblasts (6). These metaplastic cells lay down osteoid, which ultimately mineralizes and evolves into mature bone (6).

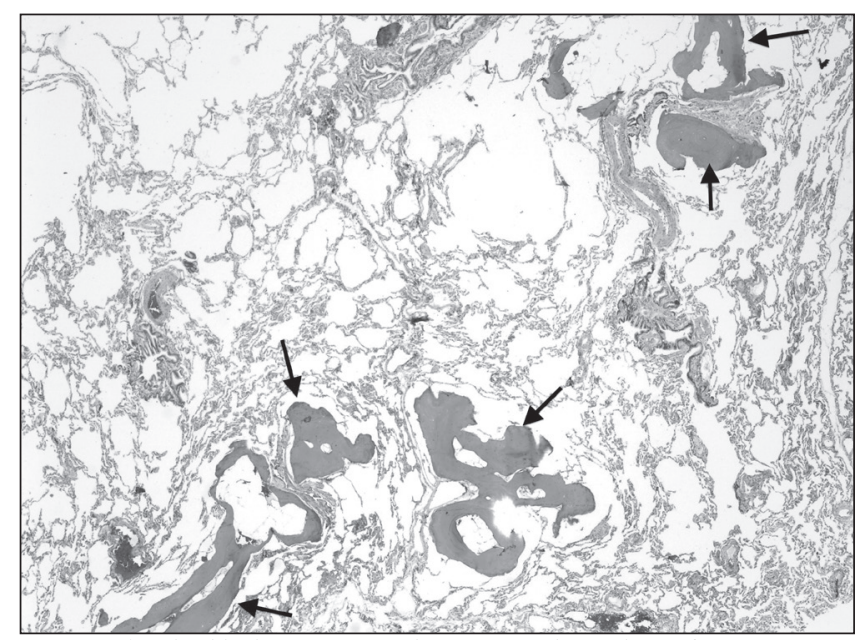

Figure 3) The black arrows on the image indicate bone fragments containing marrow

The diagnosis of DPO is generally made using high-resolution CT and lung biopsy. On CT images, nodular DPO typically presents as densely calcified nodules $1 \mathrm{~mm}$ to $5 \mathrm{~mm}$ in size, with occasional punctate or miliary calcifications (7). The dendriform pattern manifests as fine linear branching opacities in the bibasilar and subpleural lung zones (8). The CT bone windows are more sensitive at ruling out false positives and have been correlated with positive histopathological findings of DPO (8).

On biopsy, dendriform ossification manifests as interstitial branching spicules of bone and marrow elements that may protrude into the alveoli and follows interstitial fibrosis (5). The nodular circumscribed form is characterized by lamellar deposits of calcified osteoid material situated within the alveolar spaces, often without marrow elements on biopsy (5).

DPO also manifests as a restrictive pattern with an impaired diffusion capacity on pulmonary function tests $(2,6,8)$. Pulmonary ossification may also be detected using $99 \mathrm{mTc}$-medronate nuclear scans $(2,6,8)$. There are no laboratory findings of diagnostic value in diagnosing DPO $(5,7)$. The condition may slowly progress over time or may remain stable.

DPO is rarely diagnosed due to lack of awareness of this entity, associated diseases and underappreciated radiographic findings (6). Achieving an accurate and timely diagnosis will enhance knowledge of pathogenesis, disease course, lead to more effective treatments and avoid erroneous treatments due to misdiagnosis.

There is no cure for DPO. Corticosteroids, calcium-binding drugs and low-calcium diets have no discernable benefit, although they have not been systematically evaluated, and the role of bisphosphonates remains to be determined (5). As the population ages and the prevalence of chronic lung disease increases, it is likely that physicians will encounter more cases of DPO.

\section{Post-test}

- What is the etiology of pulmonary ossification syndrome?

$\mathrm{DPO}$ can be idiopathic or can be caused by a variety of pulmonary diseases such as amyloidosis, metastases, pulmonary fibrosis, cardiac causes such as mitral stenosis and left ventricular failure. It can also be caused by chronic thromboembolic hypertension.

- What is the pathogenesis by which chronic thromboembolic pulmonary hypertension causes pulmonary ossification syndrome?

Tissue injury is the most important provoking factor such that, in an alkaline environment, it initiates the precipitation of calcium salts, enables alkaline phosphatase activity and activates profibrogenic cytokines that transforms fibroblast into osteoblasts. 


\section{REFERENCES}

1. Luschka H. Ramified ossification of the pulmonary parenchyma. Wirchows Arch 1856;10:500-5.

2. Konoglou M, Zarogoulidis P, Baliaka A. Lung ossification: An orphan disease. J Thorac Dis 2013:5:101-4.

3. Lara JF, Catroppo JF, Kim DU, da Costa D. Dendriform pulmonary ossification, a form of diffuse pulmonary ossification: Report of a 26-year autopsy experience. Arch Pathol Lab Med 2005;129:348-53.

4. Mathai S, Schwarz M, Ellis J. Extensive diffuse pulmonary ossification after acute respiratory distress syndrome. Am J Respir Crit Care Med 2008;187:890.
5. Peros-Golubicic T, Tekavec-Trkanjec J. Diffuse pulmonary ossification: An unusual interstitial lung disease. Curr Opin Pulm Med 2008;14:488-92.

6. Kanne JP, Godwin JD, Takasugi JE, et al. Diffuse pulmonary ossification. J Thorac Imag 2004;19:98-102.

7. Woolley K, Stark P. Pulmonary parenchymal manifestations of mitral valve disease. Radiographics 1999;19:965-72.

8. Kim T, Han J, Chung M, et al. Disseminated dendriform pulmonary ossification associated with usual interstitial pneumonia: Incidence and thin-section CT-pathologic correlation. Eur Radiol 2005;15:1581-5. 


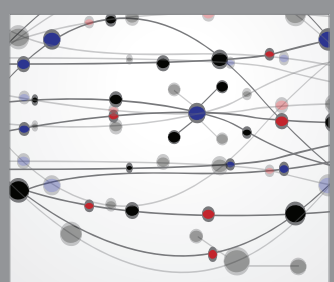

The Scientific World Journal
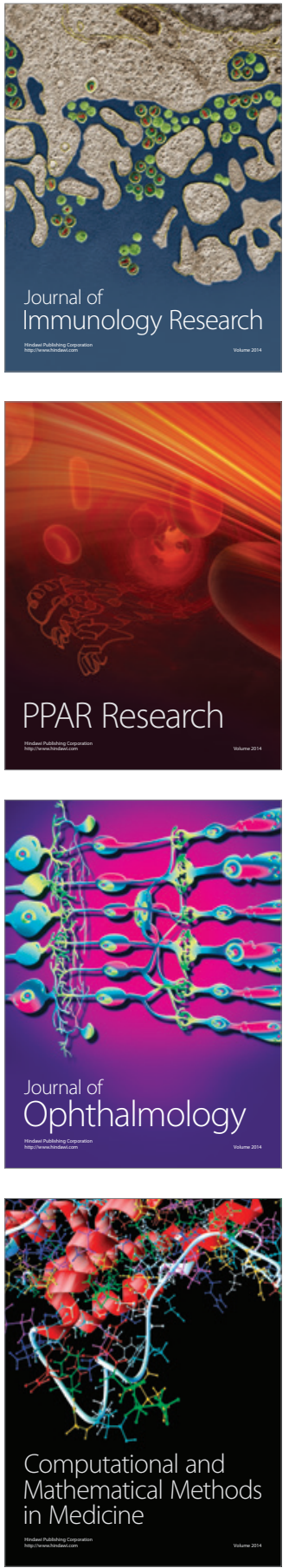

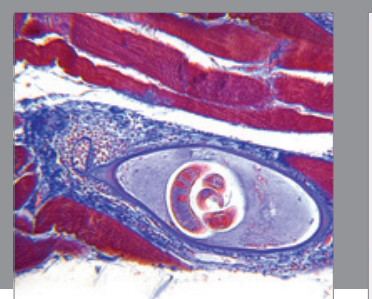

Gastroenterology Research and Practice

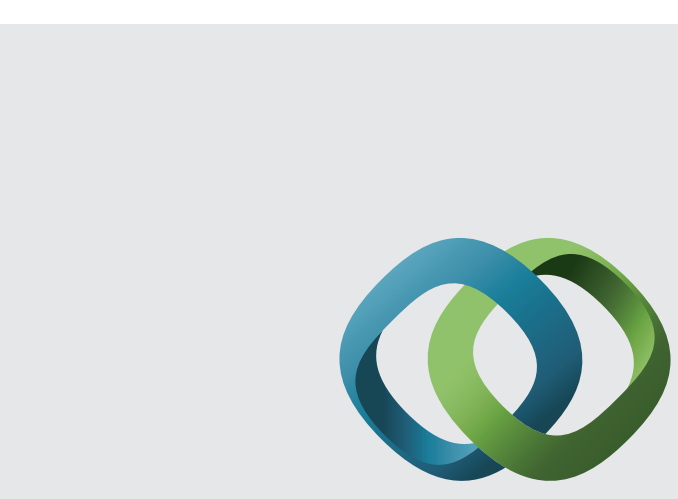

\section{Hindawi}

Submit your manuscripts at

http://www.hindawi.com
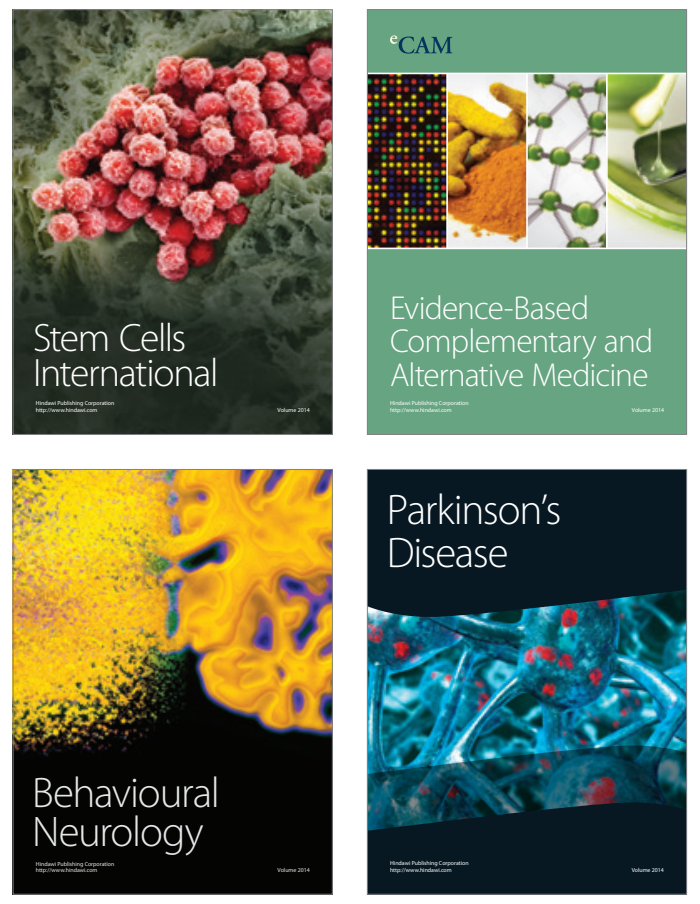
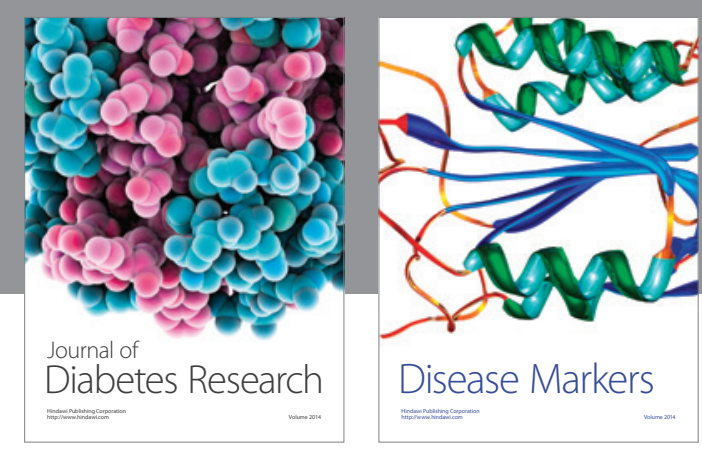

Disease Markers
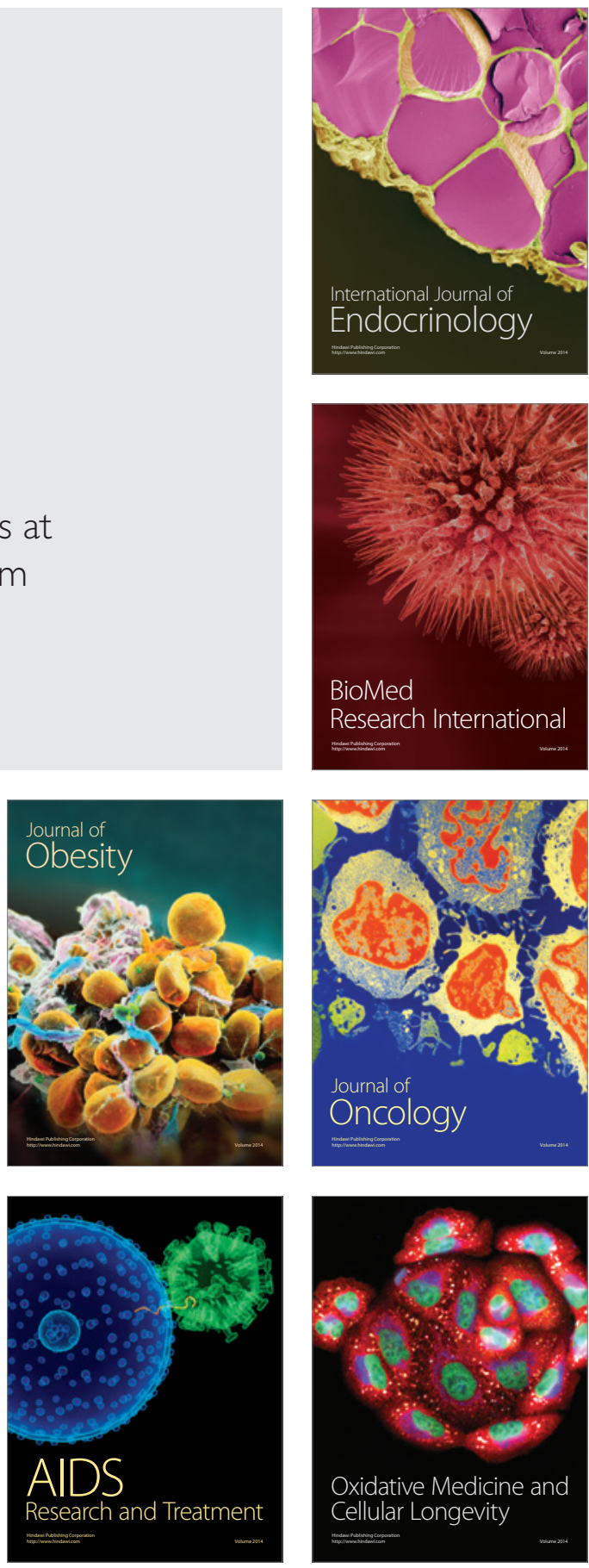